\title{
Combining Ability Analysis for Earliness, Yield and Quality Components in Tomato (Solanum lycopersicum L.)
}

\author{
Rajneesh Srivastava $^{1 *}$, V.M. Prasad ${ }^{1}$, Shailesh Marker $^{2}$, \\ Balaji Vikram $^{1}$ and Vijay Bahadur ${ }^{1}$
}

${ }^{1}$ Department of Horticulture, Naini Agricultural Institute, Sam Higginbottom University of Agriculture, Technology and Sciences, Allahabad-211007 (U.P.) India

${ }^{2}$ Sam Higginbottom University of Agriculture, Technology and Sciences, Allahabad -211007 (U.P.) India

*Corresponding author

\begin{abstract}
A B S T R A C T
Keywords

Combining ability,

Line $\times$ Tester,

Tomato, Hybrid

Article Info

Accepted:

10 June 2019

Available Online:

10 July 2019

To estimate the combining ability, fourteen tomato genotypes were crossed in Line $\times$ Tester fashion to develop 33 hybrids, which along with their parents and check were evaluated for earliness, fruit yield and quality of tomato to know extent of combining ability for the same. The lines P-7 (-4.990) and P-8 (-2.990) were found to be the best general combiner with significant highest GCA (General Combining Ability) in desirable direction for days to $50 \%$ flowering while P-4, for days to first ripening (-6.47) and P-6 (170.80) for number of fruits per plant was best general combiner. Line P3 exhibited significant GCA in desirable positive direction for average ten fruit weight (11.08), total soluble solid (0.60) and fruit texture (0.76). In case of yield per plant the lines P-10 (0.61) and P-11 (0.43) showed positive significant GCA while, P-5 for flesh thickness and to be consider as good general combiner. The tester P-13 (mutant) was a best general combiner for traits like days to first ripening (-4.77), numbers of fruits per plant (127.60) and Total soluble solid (0.27), whereas, P-12 was found best general combiner for numbers of fruits per plant (126.15) and fruit texture (0.14). Among the crosses, cross P3 x P12 (-5.10) showed the maximum desired negative SCA followed by $\mathrm{P} 1 \times \mathrm{P} 14$ (-4.01) for days to 50\% flowering. In the respect of days to first ripening the cross P4 x P13 was noted as desired negative significant SCA (-3.32). The crosses viz., P-4 $\times$ P-14 (9.74), P-1 $\times$ P14 (6.71) and P-7 $\times$ P-12 (6.52) were found to be superior specific combiners for fruit yield per plant. Cross P-8 $\times$ P-14 was a superior specific combiner for fruit quality traits like flesh thickness (0.75), total soluble solid (1.06) and texture (0.28). On the basis of superior GCA and SCA for earliness, yield and quality of the crop, suitable genotypes may be identified which can be used in further breeding programs.
\end{abstract}

\section{Introduction}

Tomatoes, aside from being tasty, are very healthy as they are known as a power house of nutrition and also popular as "protective food". In many countries it is considered as "poor man's orange" because of its attractive appearance and nutritive value. Tomato plays an important role in the economy of the farmers of India. Potato (28.9\%), tomato $(11.3 \%)$, onion $(10.3 \%)$ and brinjal $(8.1 \%)$ are the 4 major vegetables contributing $58.6 \%$ of total vegetable production in our country. India is second largest producer of tomato 
after china with $87.9 \mathrm{~m}$ ha which share 18.8 percent of total area under tomato in the world with production of $1822.66 \mathrm{M} \mathrm{T}$ which share $11.2 \%$ of total tomato production in world with productivity 20.7 t/ha (NHB Data Base 2013). Although the total cultivated area and production of tomato in our country have increased gradually over the last few years but the productivity is still very low (1795 q/ha) compared to the average of the world yield (2480 q/ha) (FAOSTAT, 2008).

The theory of specific combining ability (SCA) and general combining ability (GCA) established by Sprague and Tatum (1942) is one of the powerful tools available to estimate the combining ability effects and aids in selecting the desirable parents and crosses for the exploitation of heterosis (Sarker et al., 2002; Rashid et al., 2007). General combining ability (GCA) is attributed to additive gene effects and additive $\times$ additive epistasis and is theoretically fixable. On the other hand, specific combining ability attributable to nonadditive gene action may be due to dominance or epistasis or both and is non-fixable. Line $x$ Tester analysis is a useful tool for preliminary evaluation of genetic stock for use in hybridization programme with a view to identify good combiners. Considering this, an investigation was undertaken to identify the best parental combination having early and high yield with TLCV resistant by using the line $\times$ tester crossing method.

\section{Materials and Methods}

The experimental site was at ICAR-Indian Institute of Vegetable Research, Varanasi situated approximately $20 \mathrm{~km}$ south-west of Varanasi at the bank of Ganga River and exists between $82.52^{\circ} \mathrm{E}$ longitude and $25.10^{\circ} \mathrm{N}$ latitude at an elevation of $128.93 \mathrm{~m}$ from mean sea level (MSL). The selected piece of land was homogenous in fertility status and uniform in textural build up. The soil of experimental field was the sandy loam in texture and sufficiently well drained. Chemically the soil was neutral in reaction with low organic carbon, deficient in nitrogen and medium in phosphorus and potash contents.

Fourteen tomato genotypes (Table 1) consisting germplasm, advance breeding lines with one leaf curl susceptible variety Punjab Chhuhara received from IIVR, Varanasi. The advance breeding line H-88-78-2 which is a leaf curl resistant mutant with altered inflorescence and ripening carrying Ty 3 gene was used in present investigation.

Thirty days old seedlings of each season were transplanted in RBD with three replications separately in a plot size of $4.50 \times 1.20 \mathrm{~m}$ at spacing of $60 \times 45 \mathrm{~cm}$. A standard package of practices was adopted to raise good crop. No insecticide was sprayed in order to protect white fly population facilitating tomato leaf curl virus (ToLCV) spread and maximum disease incidence.

\section{Development of crosses}

The crossing programme was designed to generate the F1's in 'lines $\times$ tester' mating design (Kempthorne, 1957). Fourteen parents were grown during winter season, 2015 in crossing block. Eleven cultivars used as lines (female parents) and three resistant testers viz., VRT 88-78-2, D-2-2-3 both carrying Ty3 gene and CLN-8-6-1 carrying Ty-2 gene were used as male parents.

The mature buds for emasculation were chosen from the female lines and emasculated carefully using forceps during the evening hours. Next morning pollen collected from the male parent's flower on and manual pollination on emasculated buds was done without causing any damage to the stigma. The pollinated buds were covered with cotton 
and labelled using crossing tags. The mature red ripe crossed fruits (F1's) were collected, seed extracted, dried in shade, packed in envelope and stored.

\section{Evaluation of hybrids}

Thirty three F1s with their 14 parents and check (hybrid from public sectors) were raised in nursery during rainy seasons, 2016 and about four weak old seedlings were transplanted in a well prepared plot size of 4.5 $\mathrm{x} 1.2 \mathrm{~m}$ at spacing of $60 \mathrm{x} 45 \mathrm{~cm}$ in RBD for field experiment, while 20 plants were maintained in field during year 2016-17 for data observations.

Good agronomical practices were applied timely to take better crop. Data of ten randomly selected plant of each genotypes and its crosses in each replication was recorded for different traits related to earliness and yield viz. Days to first flowering, days to 50 percent flowering, number of leaves before truss, number of fruits per plant, average ten fruit weight $(\mathrm{g})$, fruit yield per plant $(\mathrm{kg})$ and yield (q/ha) and averaged replication wise mean data was used for analysis of variance (ANOVA) at 5\% levels was done according to the model suggested by Kempthorne (1957).

\section{Results and Discussion}

Fourteen parents and their thirty three crosses were subjected to estimate the combining abilities (GCA and SCA) following the procedure proposed by Griffing (1956). The analysis of variances of combining ability was done for the eight characters in tomato. The results are presented in Table 2. The GCA and SCA variances were highly significant for all the characters under studied. The GCA variances were higher and prominent than SCA variances for all the characters under study. The estimates of general combining ability (GCA) of the parents for various characters related to horticultural traits have been shown in Table 3 and estimates of specific combining (SCA) ability for various characters have been also shown in Table 4.

\section{General combining ability}

The general combining ability effects of parents for eight characters in consequent season are presented in Table 3. General combining ability implies capacity of a parent to produce superior progeny or otherwise when crossed with another parent (Alabi et al., 1987; Izge et al., 2007).

The negative GCA effects which indicate earliness would be considered as desirable. For the days to $50 \%$ flowering maximum significant negative GCA was observed P7 (5.0) in the followed by P8 (-3.0) and to be considered good general combiner. Among the testers, none showed significant negative GCA except P12 (-0.87) which is to be considered as good combiner (Table 5).

Regarding, days to first ripening the maximum significant negative GCA was observed in P7 (-11.14) followed by P8 (-7.81) among the line and P14 (-2.50) among the tester, respectively. The parents $\mathrm{P} 7, \mathrm{P} 8$ and $\mathrm{P} 14$ are to be considered as good combiner regarding earliness characters. Similar findings have also been observed by Hannan et al., (2007b) and Saleem et al., (2013) in their studies.

For the number of fruits per plant, all the eleven lines showed significant GCA effect except P6 and P9 and out of these, five parents showed GCA effect in desired positive direction. Highest positive effect was observed in P8 (6.92) followed by P3 (3.26), P10 (3.20), P11 (2.90) and P7 (2.72). Among the tester only P12 (4.33) showed significant positive GCA effect. In respect of average ten fruit weight all the parents showed significant GCA except line P1 and tester P14. 
Table.1 List of tomato genotypes use in present investigation

\begin{tabular}{|l|l|l|l|}
\hline S. No. & Name of genotypes & Silent features & Developed by \\
\hline P 1 & VRT-2103-1-2 & Advance breeding line & ICAR-IIVR, Varanasi \\
\hline P 2 & VRT-2103-1-4 & Advance breeding line & ICAR-IIVR, Varanasi \\
\hline P 3 & VRT-2103-1-8 & Advance breeding line & ICAR-IIVR, Varanasi \\
\hline P 4 & VRT-2103-3-6 & Advance breeding line & ICAR-IIVR, Varanasi \\
\hline P 5 & VRT-2103-6-1 & Advance breeding line & ICAR-IIVR, Varanasi \\
\hline P 6 & VRT-2102-13-2 & Advance breeding line & ICAR-IIVR, Varanasi \\
\hline P 7 & EC 538441 & Early and Joint less flower & Exotic line \\
\hline P 8 & EC 538411 & Early and Joint less flower & Exotic line \\
\hline P 9 & Punjab Chhuhara & Leaf curl susceptible variety & PAU, Ludhiana \\
\hline P 10 & Punjab Barkha Bahar- 1 & TYLCV resistant variety at field condition & PAU, Ludhiana \\
\hline P 11 & Punjab Barkha Bahar-2 & TYLCV resistant variety at field condition & PAU, Ludhiana \\
\hline P 12 & D-2-2-3 & Advance breeding line carrying Ty-3 gene & ICAR-IIVR, Varanasi \\
\hline P 13 & H-88-78-2 & $\begin{array}{l}\text { Tomato leaf curl resistant mutant, with altered } \\
\text { inflorescence and ripening }\end{array}$ & ICAR-IIVR, Varanasi \\
\hline P 14 & C-8-6-1 & Advance breeding line carrying Ty-2 gene & ICAR-IIVR, Varanasi \\
\hline
\end{tabular}

Table.2 Analysis of variance in respect of various characters for combining ability

\begin{tabular}{|l|c|c|c|c|c|c|c|c|c|}
\hline Source & DF & $\begin{array}{c}\text { Days to } \\
\mathbf{5 0 \%} \\
\text { flowering }\end{array}$ & $\begin{array}{c}\text { Days to } \\
\text { first } \\
\text { ripening }\end{array}$ & $\begin{array}{c}\text { No of } \\
\text { fruits / } \\
\text { plant }\end{array}$ & $\begin{array}{c}\text { Avg. ten } \\
\text { fruits } \\
\text { weight }\end{array}$ & $\begin{array}{c}\text { Yield / } \\
\text { plant }\end{array}$ & $\begin{array}{c}\text { Flesh } \\
\text { thickness }\end{array}$ & TSS & $\begin{array}{c}\text { Texture } \\
\text { (Fruit } \\
\text { firmness) }\end{array}$ \\
\hline Replicates & 2 & $56.11 * *$ & 0.23 & 11.52 & $6763.02 *$ & 0.23 & 0.02 & $5.472^{* * *}$ & 0.02 \\
\hline Crosses & 32 & $31.99 * * *$ & $0.52 * * *$ & $134.79 * * *$ & $85695.65 * * *$ & $0.52 * * *$ & $0.97 * * *$ & $2.52 * * *$ & $0.62 * * *$ \\
\hline Line effect & 10 & 46.70 & $0.91 *$ & $162.05 *$ & 134924.80 & $0.91 *$ & 1.17 & 2.45 & $1.06 *$ \\
\hline Tester effect & 2 & 31.28 & 0.45 & $688.44 * * *$ & $\begin{array}{c}531238.40 \\
* * * *\end{array}$ & 0.45 & 1.45 & 0.558 & 0.37 \\
\hline $\begin{array}{l}\text { Line * } \\
\text { Tester effect }\end{array}$ & 20 & $24.70 * * *$ & $0.33 * * *$ & $65.80 * * *$ & $16526.80 * * *$ & $0.33 * * *$ & $0.83 * * *$ & $2.75 * * *$ & $0.43 * * *$ \\
\hline Error & 64 & 8.76 & 0.07 & 12.13 & 2132.73 & 0.07 & 0.18 & 0.64 & 0.03 \\
\hline
\end{tabular}


Table.3 Estimation of general combing ability effect for horticultural traits in 14 parents (line $\mathrm{x}$ tester) of tomato

\begin{tabular}{|c|c|c|c|c|c|c|c|c|}
\hline $\begin{array}{l}\text { Characters } \rightarrow \\
\text { Entries } \downarrow\end{array}$ & $\begin{array}{c}\text { Days to } \\
\mathbf{5 0 \%} \\
\text { flowering }\end{array}$ & \begin{tabular}{|c} 
Days to \\
first \\
ripening
\end{tabular} & $\begin{array}{c}\text { No of } \\
\text { fruits / } \\
\text { plant }\end{array}$ & $\begin{array}{c}\text { Avg. } \\
\text { ten } \\
\text { fruits } \\
\text { weight }\end{array}$ & $\begin{array}{c}\text { Yield } \\
/ \\
\text { plant }\end{array}$ & $\begin{array}{c}\text { Flesh } \\
\text { thickness }\end{array}$ & TSS & $\begin{array}{l}\text { Texture } \\
\text { (Fruit } \\
\text { firmness) }\end{array}$ \\
\hline \multicolumn{9}{|l|}{ Line (Female) } \\
\hline P-1 & 1.455 & $-2.71 *$ & 21.17 & $\begin{array}{l}4.081 \\
*\end{array}$ & -0.13 & $-0.22 * * *$ & -0.1 & 0.04 \\
\hline P-2 & 1.455 & $\begin{array}{l}-4.68 \\
* * *\end{array}$ & $66.01 * * *$ & $\begin{array}{l}6.747 \\
* *\end{array}$ & $\begin{array}{l}-0.19 \\
*\end{array}$ & $0.27 * * *$ & $\begin{array}{l}0.21 \\
* * *\end{array}$ & $0.19 * * *$ \\
\hline P-3 & $3.343 * *$ & $3.26 * *$ & $-44.15 * *$ & $\begin{array}{l}11.081 \\
* * *\end{array}$ & 0.05 & -0.02 & $\begin{array}{l}0.60 \\
* * *\end{array}$ & $0.76 * * *$ \\
\hline P-4 & 1.343 & $\begin{array}{l}-6.47 \\
* * *\end{array}$ & $68.88 * * *$ & -0.586 & $\begin{array}{l}-0.35 \\
* * *\end{array}$ & $-0.27 * * *$ & -0.01 & $0.14 * *$ \\
\hline P-5 & 0.01 & $\begin{array}{l}-4.29 \\
* * *\end{array}$ & $84.60 * * *$ & 0.636 & -0.04 & $0.77 * * *$ & $\begin{array}{l}-0.21 \\
* *\end{array}$ & 0.01 \\
\hline P-6 & 0.566 & -2.09 & $\begin{array}{l}170.80 \\
* * *\end{array}$ & 0.97 & $\begin{array}{l}0.34 \\
* * * *\end{array}$ & $0.55 * * *$ & $\begin{array}{l}-0.35 \\
* * *\end{array}$ & $-0.16 * *$ \\
\hline P-7 & $\begin{array}{l}-4.990 \\
* * *\end{array}$ & $2.72 *$ & $\begin{array}{l}-132.75 \\
* * *\end{array}$ & $\begin{array}{l}- \\
11.141 \\
* * *\end{array}$ & $\begin{array}{l}-0.22 \\
*\end{array}$ & $-0.36 * * *$ & $\begin{array}{l}0.60 \\
* * *\end{array}$ & $-0.32 * * *$ \\
\hline P-8 & $-2.990 * *$ & $6.92 * * *$ & $\begin{array}{l}-251.33 \\
* * *\end{array}$ & $\begin{array}{l}-7.808 \\
* * *\end{array}$ & $\begin{array}{l}-0.28 \\
* *\end{array}$ & $-0.32 * * *$ & $\begin{array}{l}0.31 \\
* * *\end{array}$ & -0.06 \\
\hline P-9 & 0.455 & 1.26 & $\begin{array}{l}-111.44 \\
* * *\end{array}$ & -2.586 & $\begin{array}{l}-0.21 \\
*\end{array}$ & $0.31 * * *$ & -0.11 & 0.03 \\
\hline P-10 & -0.545 & $3.20 * *$ & $82.28 * * *$ & -1.475 & $\begin{array}{l}0.61 \\
* * * *\end{array}$ & $-0.23 * * *$ & $\begin{array}{l}-0.28 \\
* * *\end{array}$ & $-0.52 * * *$ \\
\hline P-11 & -0.101 & $2.90 *$ & $45.92 * *$ & 0.081 & $\begin{array}{l}0.43 \\
* * * *\end{array}$ & $-0.49 * * *$ & $\begin{array}{l}-0.67 \\
* * *\end{array}$ & $-0.11 *$ \\
\hline SE Line & 0.9864 & 1.16 & 15.39 & 2.0004 & 0.09 & 0.06 & 0.06 & 0.05 \\
\hline SE (Gi-Gj) & 1.3949 & 1.64 & 21.77 & 2.8290 & 0.13 & 0.08 & 0.09 & 0.07 \\
\hline \multicolumn{9}{|l|}{ Tester (Male) } \\
\hline P-12 & 0.101 & $4.33 * * *$ & $-\overline{126.15^{* * * *}}$ & 0.111 & -0.04 & $0.20 * * *$ & $\begin{array}{l}-0.51 \\
* * *\end{array}$ & $-0.05 *$ \\
\hline P-13 & 0.919 & $-4.77 * * *$ & $\begin{array}{l}127.60 \\
* * *\end{array}$ & $\begin{array}{l}2.384 \\
*\end{array}$ & 0.09 & -0.00 & $\begin{array}{l}0.27 \\
* * *\end{array}$ & $0.15 * * *$ \\
\hline P-14 & -1.02 & 0.44 & -1.44 & $\begin{array}{l}-2.495 \\
*\end{array}$ & $\begin{array}{l}0.13 \\
* *\end{array}$ & $-0.20 * * *$ & $\begin{array}{l}0.24 \\
* * *\end{array}$ & $-0.09 * * *$ \\
\hline SE (GCA) & 0.5151 & 0.61 & 8.04 & 1.0447 & 0.05 & 0.03 & 0.03 & 0.02 \\
\hline SE (Gi-Gj) & 0.7285 & 0.86 & 11.37 & 1.4774 & 0.07 & 0.04 & 0.04 & 0.03 \\
\hline
\end{tabular}

* Significant at $\mathrm{P}=0.05$ level, ** Significant at $\mathrm{P}=0.01$ level, *** Significant at $\mathrm{P}=0.1$ level 
Table.4 Estimation of specific combining ability effect of line $\mathrm{x}$ tester analysis for characters related to earliness in tomato

\begin{tabular}{|c|c|c|c|c|c|c|c|c|}
\hline $\begin{array}{l}\text { Characters } \rightarrow \\
\text { Entries } \downarrow\end{array}$ & $\begin{array}{c}\text { Days to } \\
50 \% \\
\text { flowering }\end{array}$ & $\begin{array}{l}\text { Days to } \\
\text { first } \\
\text { ripening }\end{array}$ & $\begin{array}{l}\text { No of } \\
\text { fruits / } \\
\text { plant }\end{array}$ & $\begin{array}{c}\text { Avg. ten } \\
\text { fruits } \\
\text { weight }\end{array}$ & $\begin{array}{l}\text { Yield / } \\
\text { plant }\end{array}$ & $\begin{array}{c}\text { Flesh } \\
\text { thickness }\end{array}$ & TSS & $\begin{array}{c}\text { Texture } \\
\text { (Fruit } \\
\text { firmness) }\end{array}$ \\
\hline P-1 $\times$ P-12 & 0.455 & -3.976 & $-0.394 *$ & 2.889 & $-7.848 *$ & $0.348 * *$ & -0.031 & $-0.193 *$ \\
\hline P-1 $\times$ P-13 & $3.636 *$ & 0.261 & 0.062 & 0.283 & 1.137 & 0.178 & $0.659 * *$ & $0.271 * *$ \\
\hline P-1 $\times$ P-14 & $-4.091 *$ & 3.715 & $0.332 *$ & -3.172 & $6.711 *$ & $-0.526 * *$ & $-0.629 * *$ & -0.078 \\
\hline P-2 $\times$ P-12 & 3.121 & -3.642 & -0.235 & 3.222 & -4.081 & $0.239 *$ & $0.874 * *$ & 0.047 \\
\hline P-2 $\times$ P-13 & -1.364 & 2.161 & 0.15 & -0.717 & 3.627 & $0.969 * *$ & $-0.283 * *$ & 0.131 \\
\hline P-2 $\times$ P-14 & -1.758 & 1.482 & 0.084 & -2.505 & 0.454 & $-1.208 * *$ & $-0.591 * *$ & $-0.178 *$ \\
\hline P-3 $\times$ P-12 & $-5.101 * *$ & $4.880 *$ & 0.232 & -4.111 & 4.898 & $0.248 *$ & $-0.620 * *$ & -0.153 \\
\hline P-3 $\times$ P-13 & $5.414 * *$ & $-8.917 * *$ & $-0.470 * *$ & $9.949 * *$ & $-9.381 * *$ & $0.318 * *$ & $0.687 * *$ & $0.582 * *$ \\
\hline P-3 $\times$ P-14 & -0.313 & $4.037 *$ & 0.238 & -5.838 & 4.483 & $-0.566 * *$ & -0.068 & $-0.430 * *$ \\
\hline P-4 $\times$ P-12 & 1.232 & $-5.68 * *$ & $-0.380 *$ & 2.556 & $-8.52 * *$ & -0.192 & $0.417 * *$ & -0.022 \\
\hline P-4 $\times$ P-13 & 0.747 & -0.584 & -0.118 & -2.051 & -1.221 & $-0.219 *$ & $-0.623 * *$ & -0.061 \\
\hline P-4 $\times$ P-14 & -1.98 & $6.271 * *$ & $0.499 * *$ & -0.505 & $9.74 * *$ & $0.411 * *$ & 0.206 & 0.083 \\
\hline P-5 $\times$ P-12 & -0.768 & 3.869 & -0.054 & -0.667 & -2.414 & -0.154 & $0.375 * *$ & $0.205 *$ \\
\hline P-5 $\times$ P-13 & 0.747 & -1.828 & -0.168 & 0.727 & -1.873 & $-0.268 *$ & $-0.222 *$ & 0.049 \\
\hline P-5 $\times$ P-14 & 0.02 & -2.04 & 0.222 & -0.061 & 4.288 & $0.422 * *$ & -0.153 & $-0.254 * *$ \\
\hline P-6 $\times$ P-12 & 0.343 & 0.635 & 0.201 & -1.333 & 3.551 & -0.032 & $0.591 * *$ & $0.246 * *$ \\
\hline P-6 $\times$ P-13 & -2.141 & -1.628 & -0.211 & 0.727 & -3.971 & 0.161 & -0.065 & $-0.347 * *$ \\
\hline P-6 $\times$ P-14 & 1.798 & 0.993 & 0.01 & 0.606 & 0.42 & -0.129 & $-0.526 * *$ & 0.101 \\
\hline P-7× P-12 & -0.434 & $4.691 *$ & 0.291 & 2.111 & $6.520 *$ & $-0.536 * *$ & -0.014 & -0.058 \\
\hline P-7× P-13 & -2.919 & -1.839 & 0.139 & -1.828 & 1.938 & 0.147 & -0.187 & -0.028 \\
\hline P-7× P-14 & 3.354 & -2.852 & $-0.430 * *$ & -0.283 & $-8.47 * *$ & $0.390 * *$ & 0.201 & 0.086 \\
\hline P-8 $\times$ P-12 & 2.566 & -0.542 & 0.241 & 0.444 & 5.249 & -0.121 & -0.012 & 0.142 \\
\hline P-8 $\times$ P-13 & -2.586 & $6.694 * *$ & 0.083 & -3.495 & 1.013 & $-0.631 * *$ & $-1.052 * *$ & $-0.421 * *$ \\
\hline P-8 $\times$ P-14 & 0.02 & $-6.15 * *$ & $-0.323 *$ & 3.051 & $-6.262 *$ & $0.752 * *$ & $1.064 * *$ & $0.280 * *$ \\
\hline P-9x P-12 & -0.545 & -2.454 & 0.181 & -0.111 & 4.258 & $0.256 *$ & $-1.925 * *$ & $-0.246 * *$ \\
\hline P-9x P-13 & -1.697 & 1.783 & 0.139 & 2.283 & 0.675 & $-0.294 * *$ & $0.852 * *$ & 0.111 \\
\hline P-9x P-14 & 2.242 & 0.671 & $-0.320 *$ & -2.172 & -4.933 & 0.038 & $1.074 * *$ & 0.135 \\
\hline P-10 $\times$ P-12 & -0.212 & -1.12 & -0.261 & -0.889 & -4.134 & $0.266 *$ & $0.900 * *$ & 0.042 \\
\hline P-10 $\times$ P-13 & 1.303 & 2.616 & 0.224 & -1.828 & 3.623 & $-0.704 * *$ & $-0.566 * *$ & $-0.305 * *$ \\
\hline P-10 $\times$ P-14 & -1.091 & -1.496 & 0.038 & 2.717 & 0.511 & $0.438 * *$ & $-0.334 * *$ & $0.263 * *$ \\
\hline P-11 $\times$ P-12 & -0.657 & 3.346 & 0.179 & -4.111 & 2.523 & $-0.321 * *$ & $-0.555 * *$ & -0.011 \\
\hline P-11 $\times$ P-13 & -1.141 & 1.283 & 0.17 & -4.051 & 4.434 & $0.342 * *$ & $0.798 * *$ & 0.02 \\
\hline P-11 $\times$ P-14 & 1.798 & $-4.629 *$ & $-0.349 *$ & $8.162 *$ & $-6.958 *$ & -0.022 & $-0.243 *$ & -0.009 \\
\hline $\mathrm{SE} \pm(\mathrm{Sij})$ & 1.7084 & 2.0106 & 0.1558 & 3.4648 & 3.0598 & 0.1019 & 0.1059 & 0.0834 \\
\hline SE \pm (Sij-Sik) & 4.8322 & 5.6868 & 0.4408 & 9.8000 & 8.6545 & 0.2882 & 0.2996 & 0.2359 \\
\hline
\end{tabular}

* Significant at $\mathrm{P}=0.05$ level, ** Significant at $\mathrm{P}=0.01$ level, *** Significant at $\mathrm{P}=0.1$ level 
Table.5 Three best general combiner and specific combiner for different traits in both seasons

\begin{tabular}{|c|c|c|c|}
\hline S.N. & Traits & Parents & Crosses \\
\hline \multirow[t]{3}{*}{1} & Days to $50 \%$ flowering & P-7 (-4.990) & $\mathrm{P}-3 \times \mathrm{P}-12(-5.10)$ \\
\hline & & P-8 (-2.990) & $\mathrm{P}-1 \times \mathrm{P}-14(-4.09)$ \\
\hline & & P-10 (-0.545) & $\mathrm{P}-7 \times \mathrm{P}-13(-2.92)$ \\
\hline \multirow[t]{3}{*}{2} & Days to first ripening & P-4 (-6.47) & $\mathrm{P}-3 \times \mathrm{P}-13(-8.91)$ \\
\hline & & P-13 (-4.77) & $\mathrm{P}-8 \times \mathrm{P}-14(-6.15)$ \\
\hline & & P-2 (4.68) & $\mathrm{P}-4 \times \mathrm{P}-12(-5.68)$ \\
\hline \multirow[t]{3}{*}{3} & No. of fruits per plant & P-6 (170.80) & $\mathrm{P}-4 \times \mathrm{P}-14(0.50)$ \\
\hline & & P-13 (127.60) & $\mathrm{P}-1 \times \mathrm{P}-14(0.33)$ \\
\hline & & P-12 (126.15) & $\mathrm{P}-7 \times \mathrm{P}-12(0.29)$ \\
\hline \multirow[t]{3}{*}{4} & Avg. ten fruit weight & P-3 (11.08) & P-3× P-13 (9.95) \\
\hline & & P2 (6.75) & $\mathrm{P}-11 \times \mathrm{P}-14(8.16)$ \\
\hline & & P-1 (4.81) & $\mathrm{P}-2 \times \mathrm{P}-12(3.22)$ \\
\hline \multirow[t]{3}{*}{5} & Fruit yield per plant & P-10 (0.61) & P-4× P-14 (9.74), \\
\hline & & P-11 (0.43) & $\mathrm{P}-1 \times \mathrm{P}-14(6.71)$ \\
\hline & & P-14 (0.13) & $\mathrm{P}-7 \times \mathrm{P}-12(6.52)$ \\
\hline \multirow[t]{3}{*}{6} & Flesh Thickness & P-5 (0.77) & P-2x P-13 (0.97) \\
\hline & & P-2 (0.27) & $\mathrm{P}-8 \times \mathrm{P}-14(0.75)$ \\
\hline & & P-12 (0.20) & $\mathrm{P}-10 \times \mathrm{P}-14(0.44)$ \\
\hline \multirow[t]{3}{*}{7} & Total soluble solid & $\mathrm{P}-3(0.60)$ & $\mathrm{P}-9 \times \mathrm{P}-14(1.07)$ \\
\hline & & P-7 (0.60) & $\mathrm{P}-8 \times \mathrm{P}-14(1.06)$ \\
\hline & & P-13 (0.27) & $\mathrm{P}-10 \times \mathrm{P}-12(0.90)$ \\
\hline \multirow[t]{3}{*}{8} & Texture (Fruit firmness) & P-3 (0.76) & $\mathrm{P}-3 \times \mathrm{P}-13(0.58)$ \\
\hline & & $\mathrm{P}-2(0.19)$ & $\mathrm{P}-8 \times \mathrm{P}-14(0.28)$ \\
\hline & & $\mathrm{P}-12(0.14)$ & $\mathrm{P}-6 \times \mathrm{P}-12(0.25)$ \\
\hline
\end{tabular}

Among these, line P6 (170.80), P5 (84.6), P10 (82.28), P4 (68.88), P2 (66.01) and tester P11 (45.92) exhibited GCA in positive direction and are adjudged as good general combiners. For the Yield per plant among the lines, P10 (0.61) and P11 (0.43) exhibited highly significant and positive GCA effects followed by P6 (0.34) are to be considered as good combiner. However, tester P14 found to be good combiner with significant positive value of GCA (0.13). Among the lines, P9, P6 and tester P14 (0.20) exhibited significantly positive GCA effects of 0.52 and 0.41 respectively and thus were found to be good general combiners for flesh thickness. In the case of TSS, lines P5, P8 and P2 showed positive significant GCA effects of $0.77,0.69$ and 0.55 respectively and are considered to be good general combiners none of the tester showed significant GCA effect. In respect of fruit texture, among the lines, P3 (0.77), P1 (0.35) and P8 (0.28) and one tester namely P12 (0.08) were good general combiner for expression of the trait as these lines exhibited significant positive GCA.

\section{Specific combining ability}

Out of thirty three tested crosses, sixteen crosses showed negative SCA for days to $50 \%$ flowering which is desire characteristic for earliness. Among the crosses, cross P3 x P12 (-5.10) showed the maximum desired negative SCA followed by P1 x P14 (-4.01). 
In respect of days to first ripening the cross P4 x P13 was noted as desired negative significant SCA (-3.32). For the number of fruits per plant, SCA effects ranged from $8.917(\mathrm{P} 3 \times \mathrm{P} 13)$ to $6.694(\mathrm{P} 8 \mathrm{X} \mathrm{P} 13)$. The significant positive SCA effects obtained in five crosses and high magnitude of positive significant SCA effects was exhibited by P8 $\times$ P13 (6.694) followed by P4 x P14 (6.271), P3 x P12 (4.880), P7 x P12 (4.691), and P3 x P14 (4.037). These crosses were derived from good $\mathrm{x}$ poor, medium $\mathrm{x}$ poor, good $\mathrm{x}$ medium and good $\mathrm{x}$ poor general combining lines, respectively. These results are in conformity with Sharma et al., (2006), Pandey et al., (2006), Sharma and Ahmed et al., (2009), Singh et al., (2010) and Farzane et al., (2012).

In respect of average ten fruit weight nine crosses showed significant SCA effects and out of these, two crosses P4 $\times$ P14 (0.499) and P1 $\times$ P14 (0.33) showed SCA effects in positive direction which is desirable for average ten fruit weight involving good $x$ medium, good $\mathrm{x}$ poor, medium $\mathrm{x}$ good and good $\mathrm{x}$ medium general combiners, respectively. These results find support from Dharmatti et al., (2001), Gaikwad et al., (2002), Ahmed et al., (2009), Sharma and Sharma (2010), Singh and Asati (2011) and Farzane et al., (2012). For the Yield per plant among the crosses the best specific cross combinations with significant positive SCA effects which indicates good specific combining ability for fruit yield per plant were P4 × P14 (9.74), P1 × P14 (6.71), P7 × P12 (6.52) while, four crosses recorded significantly negative SCA effects ranged from $-9.38(\mathrm{P} 3 \times \mathrm{P} 13)$ to $-6.26(\mathrm{P} 8 \times \mathrm{P} 14)$ and thus considered to be poor specific cross combinations for fruit yield per plant.

These results find support from Singh et al., (2010), Virupannavar et al., (2010), Singh and Asati (2011) Saleem et al., (2013) and Aminu and Mala (2015).
Total soluble solids have been recognized as the most desirable attribute for processing (Shipton, 1960). Among the crosses, significant positive SCA effects for this trait was noticed in two crosses i.e. P7 $\times$ P14 (1.19) and P5 $\times$ P14 (1.01) and considered to be good specific combiners for TSS which are suitable for processing industry. Positive SCA effects were desirable for fruit texture. Four crosses were found superior, P3 $\times$ P13 (1.017), P8 × P13 (0.51), P2 × P14 (0.34) and $\mathrm{P} 1 \times \mathrm{P} 14(0.26)$ for this characters in the first season. Similarly, in the second season among the crosses, significant positive SCA effects were recorded in five crosses ranging from $0.205(\mathrm{P} 5 \times \mathrm{P} 12)$ to $0.58(\mathrm{P} 3 \times \mathrm{P} 13)$ and to be considered as good specific combiner. These results were in accordance with Patil (2003) and Mallangoud (2005).

The negative GCA effects which indicate earliness would be considered as desirable. The lines P7, P8 and testers P14 and P12 are to be considered as good combiner regarding both characters days to $50 \%$ flowering and days to first ripening for earliness. Regarding to yield characters the lines P10, P11 and P7 and tester P12 showed significant positive GCA effect for the number of fruits per plant, line P6, P5, P10, P4, P2 and P11 for average ten fruit weight and the line P10 and P11 and tester P14 for the yield per plant. On the basis of overall performances all these parents are adjudged as good general combiners for relative yield traits. Among the lines, P9, P6 and tester P14 exhibited significantly positive GCA effects of and thus were found to be good general combiners for flesh thickness. Among the lines, P5, P8 and P2 Two tester viz., P13 and P14 are considered to be good general combiners for TSS. In respect of fruit texture, among the lines, P3, P1 and P8 and tester P12 were good general combiner for expression of the fruit texture as these lines exhibited significant positive GCA effects and these are indicating as good combiner. The 
cross $\mathrm{P} 3 \times \mathrm{P} 12$ showed the maximum desired negative SCA followed by $\mathrm{P} 1 \times \mathrm{P} 14$ for days to $50 \%$ flowering. In respect of days to first ripening the cross $\mathrm{P} 4 \times \mathrm{P} 13$ was noted as desired negative significant SCA. For the number of fruits per plant, the significant positive SCA was exhibited by $\mathrm{P} 8 \times \mathrm{P} 13$ followed by $\mathrm{P} 4 \times \mathrm{P} 14, \mathrm{P} 3 \times \mathrm{P} 12, \mathrm{P} 7 \times \mathrm{P} 12$, and $\mathrm{P} 3 \times \mathrm{P} 14$. The crosses $\mathrm{P} 4 \times \mathrm{P} 14$ and $\mathrm{P} 1 \times$ P14 showed SCA effects in positive direction in respect of average ten fruit weight and Yield per plant. Among the crosses the best specific cross combinations were $\mathrm{P} 4 \times \mathrm{P} 14$ (9.74), P1 × P14 (6.71), P7 × P12 (6.52). Among the crosses, significant positive SCA effects for this trait was noticed in two crosses i.e. P7 $\times$ P14 (1.19) and P5 $\times$ P14 (1.01) for TSS and four crosses P3 $\times$ P13 (1.017), P8 $\times$ $\mathrm{P} 13$ (0.51), $\mathrm{P} 2 \times \mathrm{P} 14$ (0.34) $\mathrm{P} 1 \times \mathrm{P} 14(0.26)$ were found superior for fruit texture and to be considered as good specific combiner.

In conclusion, the lines P-7 and P-8 were found to be the best general for days to $50 \%$ flowering while P-4, for days to first ripening and P-6 or number of fruits per plant was best general combiner. Line P-3 exhibited significant GCA in desirable positive direction for average ten fruit weight, total soluble solid and fruit texture). In case of yield per plant the lines $\mathrm{P}-10$ and $\mathrm{P}-11$ showed positive significant GCA while, P-5 for flesh thickness and to be considered as good general combiner. The tester P-13 (mutant) was a best general combiner for traits like days to first ripening, numbers of fruits per plant and Total soluble solid ,whereas, P-12 was found best general combiner for numbers of fruits per plant and fruit texture. Among the crosses, cross P3 x P12 showed the maximum desired negative SCA followed by P1 x P14 for days to $50 \%$ flowering. In the respect of days to first ripening the cross $\mathrm{P} 4 \times \mathrm{P} 13$ was noted as desired negative significant SCA. The crosses viz., $\mathrm{P}-4 \times \mathrm{P}-14, \mathrm{P}-1 \times \mathrm{P}-14$ and $\mathrm{P}-7 \times$
P-12 were found to be superior specific combiners for fruit yield per plant. Cross P-8 $\times$ P-14 was a superior specific combiner for fruit quality traits like flesh thickness, total soluble solid and fruit texture. On the basis of superior GCA and SCA for earliness, yield and quality of the crop, suitable genotypes may be identified which can be used in further breeding programs.

\section{References}

Ahmed, S., Quamruzzaman, A.K.M. and Uddin, M.N., 2011. Estimate of heterosis in tomato (Solanum lycopersicum L.). Bangladesh Journal Agricultural Research. 36 (3): 521527.

Alabi, S.O., Obilana, A.B. and Nwasike, C.C., 1987. Gene Action and Combining Ability for Quantitative Characters in Upland Cotton. Samaru Journal of Agricultural Research. 5: (1-2): 59-64.

Aminu, D. and Mala, K.K., 2015. Combining Ability Estimates for Tomato (Lycopersicon lycopersicum (L.) H. Karst) Resistance to Nematode (Meloidogyne spp.) in the Sudan Savannah of Nigeria. Journal of Agricultural Economics, Environment and Social Sciences. 1(1):4045.

Dharmatti, P.R, Mandalgeri, B. and Band, P.G., 2001. Combining ability studies in summer tomato. Karnataka Journal of Agricultural Research. 14(2): 417-422.

F.A.O. 2000. Statistical Division, http//faostat. Fao.org. pp16-21.

Farzane, A., Nemati, H., Arouiee, H., Kakhki, A.M. and Vahdati, N., 2012. The estimate of combining ability and heterosis for yield and yield components in tomato (Lycopersicon esculentum Mill.). Journal of Biology and Environmental Sciences. 6 (17): 129-134.

Gaikwad, S.P., Raijadhav, S.B., Dumbre, A.D. and Bhor, T.J., 2002. Combining ability analysis in tomato by use of line $\mathrm{x}$ tester technique. Journal of Maharastra Agricultural University. 27(3): 308-310.

Griffing, B., 1956. Concept of general and specific combining ability in relation to 
diallel crossing system. Australian Journal of Biological Science. 9: 463-493.

Hannan, M.M, Manosh, K.B., Mohammad, B.A., Monzur, H., and Rafiul, I., 2007b. Combining ability analysis of yield and yield components in tomato (Lycopersicum esculentum Mill.). Turkish Journal of Botany. 31: 559-563.

Izge, A.U. and Garba, Y.M., 2012. Combining Ability for Fruit Worm Resistance in some commercially grown tomatoes in parts of North Eastern Nigeria. International Journal of Agricultural Sciences. 2(8): 240244.

Kempthorne, O. 1957. An Introduction to genetic Statistic. John Wiley and Sons, Inc. New York. pp: 208-223.

Mallangowda, S., 2005. Studies on double crosses involving potential purple brinjal hybrids. M. Sc. (Agri.) Thesis, Uni. Agric. Sci. Dharwad (India).

NHB, Data Base, 2013.

Pandey, S.K, Dixit, J., Pathak, V.N. and Singh, P.K., 2006. Line $x$ Tester analysis for yield and quality characters in tomato (Solanum lycopersicon (Mill.). Vegetable Science. 33(1): 13-17.

Patil, V.S., 2003. Studies on double crosses involving potential tomato hybrids, M.Sc. (Agri.) Thesis, Uni. Agric. Sci. Dharwad (India).

Rashid, M., Cheema, A.A. and Ashraf, M., 2007. Line $\times$ tester analysis in basmati rice. Pakistan Journal of Botany. 39, 2035-2042.

Saleem, M.Y., Asghar, M. and Iqbal, Q., 2013. Augmented analysis for yield and some yield components in tomato (Lycopersicon esculentum Mill.). Pakistan Journal of Botany. 45(1): 215-218.

Sarker, U., Biswas, P.S., Prasad, B. and Khaleque, M.M.A., 2002. Heterosis and genetic analysis in rice hybrid. Pakistan Journal of Biological Sciences. 5 (1): 1-5.

Sharma, D. and Sharma, H.R., 2010. Combining ability analysis for yield and other horticultural traits in tomato. Indian Journal of Horticulture. 67(3):402-405.

Sharma, J.P., Kumar, S., Singh, A.K. and Bhushan, A., 2006. Variability and inter relationship studies in tomato (Lycopersicon esculentum mill.). Journal of Research, SKUVAST-J. 5(1): 100-104.

Singh, A.K. and Asati, B.S., 2011. Combining ability and heterosis studies in tomato under bacterial wilt condition. Bangladesh Journal of Agricultural Research. 36(2): 313-318.

Singh, B., Kaul, S., Kumar, D. and Kumar, V., 2010. Combining ability for yield and its contributing characters in tomato. Indian Journal of Horticulture. 67(1): 50-55.

Sprague, G.F. and Tatum, L.A., 1942. General versus specific combining ability in single crosses of corn. Journal of American Society of Agronomy. 34: 923-932.

Virupannavar, H., Dharmatti, P.R., Kumar, K.H.Y. and Sogalad, A., 2010. Estimation of heterosis for bacterial wilt resistance in tomato. Asian Journal of Horticulture. 5(1): 40-43.

\section{How to cite this article:}

Rajneesh Srivastava, V.M. Prasad, Shailesh Marker, Balaji Vikram and Vijay Bahadur. 2019. Combining Ability Analysis for Earliness, Yield and Quality Components in Tomato (Solanum lycopersicum L.). Int.J.Curr.Microbiol.App.Sci. 8(07): 878-887.

doi: https://doi.org/10.20546/ijcmas.2019.807.105 